\title{
Seminare / Séminaires 2013
}

\section{Praxiseröffnung/-übernahme}

Das Seminar richtet sich an Ärztinnen und Ärzte, welche vor einer Praxiseröffnung (Einzel-/Gruppenpraxis), dem Einstieg in eine Gruppenpraxis oder vor einer Praxisübernahme stehen.

\section{Themen}

- Juristische Aspekte (Praxisbewilligung, Zulassung zur Sozialversicherung, Vertragswesen)

- Gesellschaftsformen/Ehe- und Erbrecht (Privat-/Geschäftsvermögen, Güterstand, Erbschaftsplanung)

- Praxiseinrichtung (Inneneinrichtung, Kostenberechnung)

- Praxisadministration (Leistungserfassungsund Abrechnungssysteme)

- Bewertung einer Arztpraxis Berechnung Inventarwert und Goodwill als Verhandlungsbasis)

- Finanzierung der Arztpraxis (Businessplan, Kredite, Absicherungsmöglichkeiten)

- Versicherungen/Vorsorge/Vermögen (Personen- und Sachversicherungen, Vorsorgeplanung).

\section{Sponsoren}

Die Kosten werden durch diverse Sponsoren (siehe www.fmhservices.ch) gedeckt.

\begin{tabular}{|c|c|c|}
\hline \multicolumn{3}{|c|}{ Datum } \\
\hline K05 & $\begin{array}{l}\text { Donnerstag, } \\
\text { 7. November } 2013 \\
9.00-16.30 \text { Uhr }\end{array}$ & $\begin{array}{l}\text { Basel } \\
\text { Hotel Victoria }\end{array}$ \\
\hline
\end{tabular}

\section{Praxisübergabe}

Das Seminar richtet sich an zukünftige Praxisübergeber/innen. Idealtermin: 5-10 Jahre vor geplanter Übergabe (aus steuer- und vorsorgeplanerischen Gründen).

\section{Themen}

- Juristische Aspekte (Praxisübergabevertrag, allg. Vertragswesen, Übergabe der Krankengeschichten)

- Nachfolgeplanung und Bewertung einer Arztpraxis (projektorientiertes Vorgehen in der Nachfolgeplanung, Berechnung Inventarwert und Goodwill als Verhandlungsbasis)

- Versicherungen/Vorsorge/Vermögen (Übergabe/Auflösung von Versicherungsverträgen, Pensions- und Finanzplanung)

- Steuern (Steueraspekte bei der Praxisübergabe: Optimierung der steuerlichen Auswirkungen, Liquidations- und Grundstückgewinnsteuer, Bestimmung des optimalen Übergabezeitpunktes).

\section{Sponsoren}

Die Kosten werden durch diverse Sponsoren (siehe www.fmhservices.ch) gedeckt.

\section{Datum \\ K10

$\begin{array}{lll}\text { K10 Donnerstag, } & \text { Basel } \\ \text { 14. November } 2013 & \text { Hotel Victoria } \\ \text { 13.30-18.00 Uhr } & \end{array}$

\section{Praxiscomputerworkshop}

Der Workshop richtet sich an praxiseröffnende sowie an bereits praxistätige Ärztinnen und Ärzte.

\section{Inhalt}

- Anforderungen an ein Praxisinformationssystem (Einführung)

- Evaluationsprozess (projektorientiertes Vorgehen in der Evaluation eines Praxisinformationssystems)

- Präsentation von sechs führenden Praxisinformationssystemen (Leistungserfassung, elektronisches Abrechnen unter Einbezug der TrustCenter, Agendaführung, Statistiken, Laborgeräteeinbindung, elektronische Krankengeschichte, Finanzbuchhaltungslösungen usw.).

\section{Datum \\ 15

$\begin{array}{ll}\text { Donnerstag, } & \text { Olten } \\ \text { 28. November } 2013 & \text { Stadttheater } \\ \text { 13.30-18.00 Uhr } & \end{array}$

\section{Folgende Softwareanbieter können Sie} im Workshop kennenlernen:

\section{Ärztekasse Genossenschaft, Urdorf \\ (Praxiscockpit CB)}

Standeseigene Genossenschaft seit 1964 - Im Bereich Praxisadministration bietet die Ärztekasse ganzheitliche und individuelle Lösungen, abgestimmt auf Ihre Bedürfnisse. Von der Beratung und Mithilfe bei einer Eröffnung oder Übernahme einer Praxis, Evaluation von Hardund Software, Netzwerkaufbau, Installation, Schulung bis zu Abrechnung und Eintreiben ausstehender Forderungen. Auch betriebswirtschaftlich unterstützen wir Schweizer Praxen und bieten Hand für statistische Erhebungen, z.B. für die FMH Roko oder kantonalen Verbände. Wir treiben Innovationen voran und stellen diese nach gründlicher Prüfung allen unseren Mitgliedern meist kostenlos zur Verfügung. Mit 10 Agenturen in der ganzen Schweiz sind wir kundennah. Als Anlaufstelle für wichtige und/oder dringende Anliegen wie Fragen zu Rechnungen, Support vor Ort (innert nützlicher Frist) oder als Informationszentrum. Unsere Standorte befinden sich in Basel,
Bern, Chur, Crissier, Genf, Lugano, Luzern, Neuchâtel, St.Gallen, Thônex und Zürich. Unser Gewinn ist Ihr Erfolg!

\section{Delemed AG Medical Software, Kehrsatz (pex II)}

Delemed AG entwickelt bereits seit über 20 Jahren erfolgreich Medizinsoftware für Praxen. Die Software besticht durch ihren sympathischen, effizienten, einfachen und modularen Aufbau und lässt in keiner Praxis Wünsche offen. Dank unserer Vielseitigkeit im medizinischen Umfeld sind wir Ihr optimaler Partner für die Zukunft.

\section{Kern Concept AG, Herisau AR (AESKULAP)} AESKULAP ist ein extrem effizientes Administrationsprogramm, das zusammen mit einer SQL-Datenbank höchste Verarbeitungsgeschwindigkeit, Kompaktheit und schnelle Zugriffszeiten gewährleistet. AESKULAP bietet für jedes Budget und für alle Bedürfnisse eine optimale und intelligente Lösung: Vom einfachen Abrechnungssystem mit sämtlichen Leistungserfassungsmöglichkeiten bis hin zur führenden vollstrukturierten elektronischen KG-Führung, die in der Praxis keine Wünsche offen lässt. AESKULAP ist ein modular aufgebautes und anpassbares System mit einer Vielzahl von Modulen. Unsere extrem preisgünstige Basisversion kann somit bei Bedarf jederzeit erweitert werden. AESKULAP - die intelligente Ärztesoftware für jedes Budget!

\section{HCI Solutions AG Abteilung TriaMed, Gümligen (TriaMed ${ }^{\circledR}$ )}

Die Abteilung TriaMed ${ }^{\circledR}$ von HCI Solutions, ein Unternehmen der Galenica Gruppe, entwickelt und vermarktet innovative Softwarelösungen für das Praxismanagement. Wir bieten individuelle Beratung, Soft- und Hardware, einen umfassenden Support und Schulung aus einer Hand. Die Softwarelösung TriaMed ${ }^{\circledR}$ ist für sämtliche Arten von Arztpraxen und Praxisgruppierungen geeignet. Basierend auf der neusten Technologie wurde TriaMed ${ }^{\circledR}$ von Ärzten für Ärzte entwickelt. So ist eine moderne und intuitiv bedienbare PraxismanagementLösung mit integrierter Krankengeschichte entstanden. Um den stetig wachsenden Anforderungen an eine praxisgerechte Softwarelösung gerecht zu werden, wird TriaMed ${ }^{\circledR}$ stetig weiterentwickelt.

\section{TMR Triangle Micro Research AG,}

\section{Hölstein (WinMed ${ }^{\circledR}$ )}

Die TMR AG wurde von Ärzten, Wissenschaftlern, Unternehmern und Dozenten der Uni Basel 1993 als «Spin-off»-Firma gegründet. 
Den Schwerpunkt in der Entwicklung der TMR AG bilden Anwendungen im Bereich der med. Telekommunikation, Internet sowie der mehrmandanten- und mehrplatzfähigen Ärztesoftware WinMed ${ }^{\circledast}$. WinMed ${ }^{\circledast}$ ist ein vollständiges, äusserst einfach zu bedienendes Arztpraxisinformationssystem mit integrierter Kommunikationsplattform, Bild- und Dokumentenverwaltung sowie modernster elektronischer Krankengeschichte. WinMed ${ }^{\circledast}$ wird nach ausgereifter mehrjähriger Pilotphase erfolgreich seit 1998 verkauft und zählt heute zu den meistgekauften Ärztesoftwarepaketen. Vertrieben wird WinMed ${ }^{\circledast}$ in der Deutschschweiz von TMR AG selbst und im Tessin von GFP mediconsul informatica in Massagno.

\section{Vitodata AG, Oberohringen (vitodata)}

Als unabhängiges Schweizer Familienunternehmen agiert die Vitodata seit über 30 Jahren im Gesundheitswesen mit dem Ziel, effiziente IT-Lösungen für praktizierende Ärzte und Ärztinnen zu entwickeln. Mit unserer neuen Applikation vitodata ist ein innovatives Praxisinformationssystem verfügbar. Es setzt neue Massstäbe in Design und Benutzbarkeit und ist perfekt auf die Bedürfnisse des modernen Praxisalltags zugeschnitten. Mit Hilfe der übersichtlichen Oberfläche im Outlook-Stil und der intuitiven Benutzerführung finden Sie sich schnell zurecht. Bei aufkommenden Fragen ist eine situative und z. T. audiovisuelle Hilfe verfügbar. Bei weiterführenden Anwendungsfragen und technischen Problemen ist unsere Hotline für Sie da. Verschiedene Interventionslevels erlauben eine schnelle Hilfe - auch ausserhalb des Wartungsvertrags. Mit vitodata erhalten Sie den gesamten Funktionsumfang zu einem Preis und für eine unbegrenzte Zeit. Der erstklassige Service und die innovative Roadmap bieten Ihnen höchste Investitionssicherheit. Als Alternative zum Lizenzvertrag bietet sich das ASP-Modell (Application Service Providing) an. $\mathrm{Zu}$ einem monatlichen Mietpreis erhalten Sie einen bequemen Zugang auf die bei uns abgelegte Software. So können Sie Ihre IT-Infrastruktur einfach halten - im Minimalfall reichen bereits sogenannte «Thin-Clients».

\section{Telefonseminar für MPA}

(bzw. Praxisteam)

Das Seminar richtet sich an MPAs mit telefonischem Kundenkontakt sowie Auszubildende, die zum professionellen Telefonieren angeleitet werden sollen.

\section{Themen}

- Die Medizinische Praxisassistentin als Visitenkarte der Praxis

- Image der Arztpraxis. MPAs repräsentieren die Unternehmenskultur, organisieren die Praxis und sind somit ein wesentlicher Bestandteil für den Unternehmenserfolg.

- Bedeutung des ersten Telefonkontakts

- Richtig telefonieren - eine Anleitung.

\section{Kosten}

300 CHF (inkl. sämtlicher Kursunterlagen und Verpflegungen).

Praxisteams erhalten einen Rabatt von 20\% pro Teilnehmer/in.

$\begin{array}{lll}\text { Datum } & & \\ \text { K66 } & \text { Mittwoch, } & \text { Bern } \\ & \text { 06. November } 2013 & \text { Schmiedstube } \\ \text { 09.00-16.30 Uhr } & \end{array}$

Ouverture et reprise d'un cabinet médical Le séminaire est destiné aux médecins sur le point d'ouvrir un cabinet médical (individuel ou de groupe), de joindre un cabinet de groupe ou de reprendre un cabinet existant.

\section{Contenu}

- Business plan (préparation du plan de financement et crédit d'exploitation, financement par la banque)

- Aménagement (implantation, projet et concept d'aménagement, choix du mobilier, budget)

- Estimation d'un cabinet (inventaire et goodwill)

- Administration d'un cabinet médical (dans le cabinet, par la banque)

- Assurances (toutes les assurances à l'intérieur et autour du cabinet)

- Passage du statut de salarié à celui d'indépendant

- Fiscalité

\section{Sponsors}

Les coûts sont pris en charge par divers sponsors (voir www.fmhservices.ch).

$\begin{array}{lll}\begin{array}{ll}\text { Date } \\ \text { K23 }\end{array} & \begin{array}{l}\text { Jeudi } \\ 21 \text { novembre } 2013 \\ \\ 17.00-21.30 \mathrm{~h}\end{array} & \begin{array}{l}\text { Genève } \\ \text { Crowne Plaza }\end{array}\end{array}$

Remise d'un cabinet médical

Le séminaire s'adresse aux médecins désirant remettre un cabinet médical. Idéalement 5-10 ans avant la remise prévue (pour des questions de taxation et prévoyance).

\section{Contenu}

- Aspects juridiques (autour du contrat de remise/reprise)

- Estimation d'un cabinet (inventaire et goodwill)

- Assurances (prévoyance, assurances à l'intérieur et autour du cabinet)

- Conséquences fiscales d'une remise.

\section{Sponsors}

Les coûts sont pris en charge par divers sponsors (voir www.fmhservices.ch).

\section{Date \\ K25 Jeudi 14 novembre 2013 \\ 17.00-21.30 h Genève Crowne Plaza}

\section{Anmeldung und Auskunft /}

Inscription et information

www.fmhservices.ch oder FMH Consulting Services, Cornelia Fuchs, Burghöhe 1, 6208 Oberkirch, Tel. 04192500 77, Fax 0419210586.

\section{Hinweis / Remarque}

Bei sämtlichen Seminaren, bei denen die Kosten teilweise oder gänzlich von Seminarsponsoren gedeckt werden, werden die Teilnehmeradressen den jeweiligen Sponsoren zur Verfügung gestellt. / Les adresses des participants aux séminaires dont les coûts sont couverts en partie ou totalement par des sponsors sont communiquées aux sponsors concernés.

\section{Annullierungsbedingungen /}

\section{Conditions d'annulation}

Bei Abmeldungen oder Fernbleiben werden folgende Unkostenbeiträge erhoben: / Un montant est perçu pour une absence ou une annulation. Il est de:

- 50 CHF pro Person ab 14 Tage vor Seminarbeginn / par personne dans les 15 jours avant le début du séminaire;

- 100 CHF pro Person ab 7 Tage vor Seminarbeginn oder Fernbleiben / par personne dans les 7 jours avant le début du séminaire. 